\title{
Comparison of a flexible versus a rigid breast compression paddle: pain experience, projected breast area, radiation dose and technical image quality
}

\author{
Mireille J. M. Broeders • Marloes ten Voorde • Wouter J. H. Veldkamp • Ruben E. van Engen • \\ Cary van Landsveld - Verhoeven • Machteld N. L. 't Jong - Gunneman • Jos de Win • \\ Kitty Droogh-de Greve • Ellen Paap • Gerard J. den Heeten
}

Received: 7 May 2014 / Revised: 12 August 2014 / Accepted: 28 August 2014 /Published online: 11 December 2014

(C) The Author(s) 2014. This article is published with open access at Springerlink.com

\begin{abstract}
Purpose To compare pain, projected breast area, radiation dose and image quality between flexible (FP) and rigid (RP) breast compression paddles.

Methods The study was conducted in a Dutch mammographic screening unit (288 women). To compare both paddles one additional image with RP was made, consisting of either a mediolateral-oblique (MLO) or craniocaudal-view (CC). Pain experience was scored using the Numeric Rating Scale (NRS). Projected breast
\end{abstract}

Electronic supplementary material The online version of this article (doi:10.1007/s00330-014-3422-4) contains supplementary material, which is available to authorized users.

M. J. M. Broeders $(\varangle) \cdot$ W. J. H. Veldkamp • R. E. van Engen •

C. van Landsveld - Verhoeven • M. N. L. 't Jong - Gunneman •

E. Paap · G. J. den Heeten

Dutch Reference Centre for Screening, PO Box 6873, 6503

GJ Nijmegen, The Netherlands

e-mail: m.broeders@lrcb.nl

M. J. M. Broeders

Department for Health Evidence, Radboud University Medical

Center, Nijmegen, The Netherlands

M. ten Voorde

Organization for Cancer Screening in the Eastern Part of the

Netherlands, Deventer, The Netherlands

W. J. H. Veldkamp

Department of Radiology, Leiden University Medical Center,

Leiden, The Netherlands

J. de Win • K. D.-d. Greve

Gelre Hospital Apeldoorn, Apeldoorn, The Netherlands

G. J. den Heeten

Department of Radiology, Academic Medical Centre, Amsterdam,

The Netherlands area was estimated using computer software. Radiation dose was estimated using the model by Dance. Image quality was reviewed by three radiologists and three radiographers.

Results There was no difference in pain experience between both paddles (mean difference NRS: $0.08 \pm 0.08$, $p=0.32$ ). Mean radiation dose was $4.5 \%$ lower with FP $(0.09 \pm 0.01 p=0.00)$. On MLO-images, the projected breast area was $0.79 \%$ larger with FP. Paired evaluation of image quality indicated that FP removed fibroglandular tissue from the image area and reduced contrast in the clinically relevant retroglandular area at chest wall side.

Conclusions Although FP performed slightly better in the projected breast area, it moved breast tissue from the image area at chest wall side. RP showed better contrast, especially in the retroglandular area. We therefore recommend the use of RP for standard MLO and $\mathrm{CC}$ views.

Key points

- Pain experience showed no difference between flexible and rigid breast compression paddles.

- Flexible paddles do not depict clinically relevant retroglandular areas as well.

- Flexible paddles move breast tissue from image area at the chest wall side.

- Rigid paddles depict more breast tissue and shows better contrast.

- Rigid breast compression paddles are recommended for standard mediolateral-oblique and craniocaudal views.

Keywords Mammography · Compression paddle · Performance $\cdot$ Flexible compression paddle $\cdot$ Rigid compression paddle 


\section{Introduction}

Compression of the breast in mammography is essential for reducing radiation dose, preventing motion artefacts, and obtaining flattened breast tissue with a homogeneous tissue thickness to improve the dynamic range of luminance and improve visibility by spreading structures in the breast [1]. An important disadvantage of compression is the pain and discomfort that women experience during and after the examination [2-4]. In some cases, the pain is extremely severe and those women might decide to avoid mammography. However, so far, nearly all efforts to relieve the number of complaints caused by compression have failed [5].

At the time digital mammography was implemented, socalled flexible compression paddles were introduced with the aim to improve comfort in mammography [6]. These paddles can be tilted with a hinging mechanism to adjust their position to the conic shape of the breast (see Fig. 1). Mammography units in the Netherlands are currently supplied with two different compression paddles for screening and diagnostic purposes: a flexible (FP) and a rigid compression paddle (RP). The RP remains approximately parallel to the detector during compression, whereas the FP remains parallel to the detector at first, tilts towards nipple side and ends with the highest point at thorax level. Some manufacturers endorse the use of FP, suggesting it would be less painful for the women [6], but in practice the paddles are used interchangeably.

To our knowledge, even though the design of the FP differs fundamentally from the more commonly used RP, no comprehensive study has yet been conducted to study differences in the pain experience and the overall clinical performance of these two paddles. Sardanelli et al. compared the performance of two paddles using monophasic or biphasic compression, respectively, and found the latter performed better on image quality and the amount of breast area depicted [7]. However, monophasic compression was similar to the RP, but the tilt in biphasic compression was opposite to the FP used in this study. The biphasic compression started angled downward, with the angle being progressively reduced until it was parallel to the cassette holder. A more recent paper by Hauge et al. compared the readout thickness displayed by the mammography machine to the measured thickness of the compressed breast over a range of mammography units, and found greater departures from measured thickness for flexible paddles compared to nonflexible paddles [8].

The purpose of this prospective cross-over trial is to compare pain experience, projected breast area, radiation dose and technical image quality between FP and RP.

\section{Materials and methods}

A permit for this study, equal to institutional review board approval, was obtained from the Minister of Health, Welfare and Sport under the Dutch Population Screening Act in October 2010. All women participating signed informed consent.

Study population and design

The Dutch screening program invites women aged 50 to 75 years biennially and is regionally executed by five screening organizations and 65 screening units [9]. This study was performed in one mobile screening unit of the screening program for the eastern part of the Netherlands, where standard FP was used. Each screening examination consists of two views, a mediolateral oblique (MLO) and a craniocaudal (CC) view, per breast.

Women, who were invited for a screening examination in October and November 2010, received an information leaflet about the aim of the study one week before their appointment. At the screening, the radiographer answered questions regarding the study and, if positive, asked the woman for informed consent. Women were excluded from the study if they were not of sound mind and judgment (mentally ill or mentally handicapped), if they did not speak or understand the Dutch language and if they had a breast prosthesis.

All mammograms were acquired on a dedicated mammography system, Selenia (Hologic, Danbury, Conn, USA), with $18 \times 24$ and $24 \times 29 \mathrm{~cm}$ fields of view (compression force 12.8 $\pm 1.3 \mathrm{daN}$ ). The target force for all compressions was between 12 and $20 \mathrm{daN}$, but radiographers were allowed to cease compression when the woman verbally expressed severe pain.
Fig. 1 Compression of the breast with RP (left) and FP (right). The $\mathrm{RP}$ remains approximately parallel to the detector during compression, whereas the FP remains parallel to the detector at first, tilts towards nipple side and ends with the highest point at thorax level
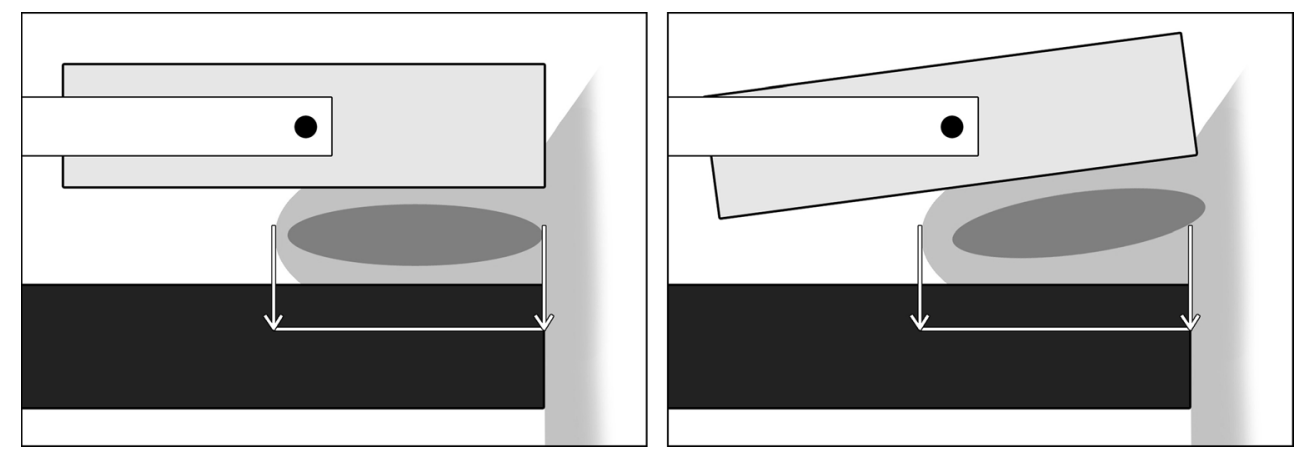
For FP and RP, the same target force for compression was used, which was registered in the DICOM-header. To enable pairwise comparisons for FP and RP for the same breast and mammographic projection, all women participating in the study received an additional image with $\mathrm{RP}$ for one out of four views, i.e., the left or right $\mathrm{MLO}$ or left or right $\mathrm{CC}$ view, with the order of paddles randomly assigned for the selected view (prospective cross-over design). To minimize differences in the performance of the screening examinations, only three experienced radiographers were involved. The radiographers were skilled in the use of both paddles. Because the differences between the paddles are obvious, the radiographers could not be blinded for the compression paddle that was used. However, before the start of the study, they received instruction about the importance of objective measurements. Also, before the start of the study, we had no expectations about the performance of the paddles.

\section{Pain experience}

Pain was assessed using a Numerical Rating Scale (NRS) and was scored by the woman directly after each compression (see Table 1). The NRS is an 11-point scale from 'no pain' to 'severe pain,' and is considered a valid instrument to measure pain [10].

\section{Projected breast area}

The length of the posterior nipple line and the projected breast area were estimated to determine which paddle resulted in the largest projected breast area projected on the images (see Table 1).

On the digital image, the length of the posterior nipple line was measured manually as a perpendicular line from the posterior film edge to the nipple (CC) or the pectoral muscle to the nipple (MLO) $[11,12]$. To check for consistency, all measurements were performed twice. The correlation between the first and second measurement was $0.995(p=0.00)$ and 0.998 ( $p=0.00)$ for FP and RP, respectively.

The projected breast area was estimated with computer software developed in MATLAB (The Mathworks Inc, Novi, MI, USA) using data from the DICOM-header for each mammogram. Two parameters were used from the DICOM-header: the pixel value of the background region in the processed images and the pixel size. The software algorithm marked all pixels above a fixed background level in the 'for presentation' mammogram as being part of the breast surface. Morphologic operations were subsequently applied to ensure that all pixels within the breast area were marked. The algorithm determined the total number of marked pixels $n$ for each image. Since the pixel area $a$ could be determined from the DICOM-header, the projected breast area could be determined ( $n \times a)$ for each image.

\section{Radiation dose}

Radiation dose was determined using the breast dosimetry model developed by Dance et al. [1, 13]. The model of Dance is widely used and validated in the scientific literature and is recommended in the European Guidelines [1]. Apart from

Table 1 Results of the posterior nipple line distance, the projected breast area, pain experience and radiation dose for FP versus RP

\begin{tabular}{|c|c|c|c|c|c|c|}
\hline & $\mathrm{n}$ & $\mathrm{FP}($ mean \pm sem $)$ & $\mathrm{RP}($ mean \pm sem $)$ & $\begin{array}{l}\text { Difference FP versus RP } \\
(\text { mean } \pm \text { sem })\end{array}$ & $\begin{array}{l}\text { Difference FP } \\
\text { versus RP ( \%) }\end{array}$ & $\mathrm{p}$ \\
\hline \multicolumn{7}{|c|}{ Posterior nipple line distance $(\mathrm{cm})$} \\
\hline $\mathrm{CC}$ view & 144 & $10.9 \pm 0.2$ & $10.9 \pm 0.2$ & $-0.01 \pm 0.03$ & $-0.12 *$ & 0.71 \\
\hline $18 \times 24 \mathrm{~cm}$ & 93 & $9.5 \pm 0.2$ & $9.5 \pm 0.2$ & $-0.02 \pm 0.04$ & -0.17 & 0.69 \\
\hline $24 \times 30 \mathrm{~cm}$ & 51 & $13.4 \pm 0.3$ & $13.5 \pm 0.3$ & $0.00 \pm 0.05$ & -0.02 & 0.95 \\
\hline MLO view & 144 & $11.3 \pm 0.2$ & $11.2 \pm 0.2$ & $0.12 \pm 0.03$ & 1.05 & 0.00 \\
\hline $18 \times 24 \mathrm{~cm}$ & 73 & $9.3 \pm 0.2$ & $9.3 \pm 0.2$ & $0.03 \pm 0.03$ & 0.31 & 0.30 \\
\hline $24 \times 30 \mathrm{~cm}$ & 71 & $13.3 \pm 0.2$ & $13.1 \pm 0.2$ & $0.21 \pm 0.04$ & 1.58 & 0.00 \\
\hline \multicolumn{7}{|l|}{ Projected breast area $\left(\mathrm{cm}^{2}\right)$} \\
\hline $\mathrm{CC}$ view & 144 & $180.0 \pm 5.7$ & $178.6 \pm 5.6$ & $1.4 \pm 0.5$ & 0.75 & 0.11 \\
\hline $18 \times 24 \mathrm{~cm}$ & 93 & $142.6 \pm 3.7$ & $142.2 \pm 3.6$ & $0.5 \pm 0.5$ & 0.32 & 0.34 \\
\hline $24 \times 30 \mathrm{~cm}$ & 51 & $248.0 \pm 8.4$ & $245.0 \pm 8.4$ & $3.0 \pm 1.2$ & 1.22 & 0.01 \\
\hline MLO view & 144 & $218.7 \pm 5.5$ & $217.0 \pm 5.3$ & $1.7 \pm 0.8$ & 0.79 & 0.03 \\
\hline $18 \times 24 \mathrm{~cm}$ & 73 & $168.7 \pm 4.2$ & $169.2 \pm 4.2$ & $-0.5 \pm 0.8$ & -0.29 & 0.51 \\
\hline $24 \times 30 \mathrm{~cm}$ & 71 & $270.1 \pm 5.5$ & $266.1 \pm 5.4$ & $4.0 \pm 1.3$ & 1.50 & 0.00 \\
\hline Pain experience (NRS scale) & 288 & $3.82 \pm 0.14$ & $3.74 \pm 0.13$ & $0.08 \pm 0.08$ & 2.06 & 0.32 \\
\hline Breast thickness (mm) & 288 & $52.18 \pm 0.74$ & $56.97 \pm 0.67$ & $-4.79 \pm 0.20$ & -8.4 & 0.00 \\
\hline Radiation dose (mGy) & 288 & $1.83 \pm 0.03$ & $1.91 \pm 0.03$ & $-0.09 \pm 0.01$ & -4.5 & 0.00 \\
\hline
\end{tabular}

* a minus sign indicates a larger nipple line distance or breast area of the RP 
general inherent uncertainties in this model, there are additional uncertainties with FP, since the model assumes a cylinder shaped, flat compressed breast and does not account for tilting paddles. With FP, the breast thickness decreases towards the nipple side, resulting in a wedge shaped breast. Due to the wedge shape of the compressed breast with FP, breast thickness is not unambiguously defined. We chose to calculate glandular dose for both compression paddles using the conversion factors for RP for the following reasons. First, the dose model assumes that the compression paddle is parallel with the breast support table (the situation with RP). Second, breast composition is a function of breast thickness in the dose model, and will thus incorrectly vary for RP and FP. Since breast composition is an inherent property of the breast, we chose to use the breast composition based on RP for dose calculation for both RP and FP. It is acknowledged that some uncertainty exists in the calculated average glandular dose levels for FP due to the fact that the dose model does not account for tilting paddles.

\section{Technical image quality}

Technical image quality was qualitatively reviewed by three radiologists and three radiographers, in two steps. The first blinded unpaired evaluation was performed by two radiologists by consensus, and by one radiographer (see Table 2). A score based on the overall impression of technical image quality of the images was obtained for both paddles and was scored on a scale from 0 to 10 . In addition, technical image quality was scored as good, sufficient or insufficient, based on the following projection criteria: area around the nipple well imaged, pectoral muscle imaged, nipple in profile, inframammary angle clearly imaged (MLO view only). Differences in image quality are more easily spotted in pairwise comparisons. A second blind paired comparison was not possible, because the differences between the two paddles were extremely clear and the observers immediately noticed which of the two images was performed with FP. Therefore, a second unblinded paired comparison by a third radiologist and two radiographers by consensus was performed using toggle mode (blinking) to evaluate if one of the two mammograms had better image quality or if they were equivalent (see Table 3). Toggle (or blink) mode alternates new and prior medical images superimposed digitally upon each other, rather than comparing them visually in the side-by-side or up-down fashion. The analyses for technical image quality were performed separately for radiographers and radiologists, because they differed in their focus in the review of technical image quality.

Statistical analysis

Differences between RP and FP in pain experience, the length of the posterior nipple line, the projected breast area, and radiation dose were compared with the paired t-test. Percent differences were estimated as follows: ((mean outcome FP*100) / mean outcome RP) - 100). Stratified analyses were performed according to paddle size, $18 \times 24 \mathrm{~cm}$ and $24 \times 29 \mathrm{~cm}$, and mammographic projection, MLO and CC. For both the unpaired and paired evaluation, differences in the criteria for technical image quality were assessed using the McNemar test. All analyses were performed using SPSS, version 19 (SPSS Inc., Chicago Ill). Two-sided $p$ values less than 0.05 were considered to indicate a statistically significant difference.

\section{Results}

In total, 698 women were invited to include 288 women, resulting in a participation rate of $41.3 \%$. Of these 698 women, 116 could not participate due to logistic reasons, 256 decided not to participate in the study, 37 needed to be excluded beforehand and one was excluded because the height of the bucky needed to be adjusted during the examination. The 37 women were excluded because they did not speak Dutch $(n=33)$, had a breast prosthesis $(n=3)$ or were not of sound mind and judgment $(n=1)$.

The 288 participants underwent one additional CC or MLO view with RP, resulting in $144 \mathrm{CC}$ and 144 MLO images obtained with RP and FP. There was no difference in mean compression force for RP compared to FP (both 12.8 daN $\pm 1.3, p=0.934)$.

Pain experience was comparable for both compression paddles (see Table 1). For FP, mean pain experience on the NRS scale was $3.82(\mathrm{sem} \pm 0.14)$ and for RP it was 3.74 ( $\mathrm{sem} \pm$ 0.13 ), resulting in a mean difference of 0.08 ( $\operatorname{sem} \pm 0.08 ; p=$ 0.32 ). In total, $34 \%$ of the women experienced moderate to severe discomfort with RP (NRS $\geq 5$ ). For FP, this proportion was $36.8 \%$, indicating a nonsignificant mean difference of 0.26 in favour of $\mathrm{RP}(p=0.087)$. The mean pain experience for both paddles tended to be somewhat higher for the MLO view (FP: $4.13 \pm 0.20$; RP: $4.02 \pm 0.19$ ), than for the $\mathrm{CC}$ view (FP: $3.50 \pm 0.20$; RP: $3.46 \pm 0.19$ ). However, in both views, the difference between FP and RP was small and not significant.

Radiation dose was determined and $0.08 \mathrm{mGy}$ lower in FP compared to RP $(1.83 \pm 0.03,1.91 \pm 0.03$ respectively; $p=$ 0.00 ), resulting in a $4.5 \%$ percentage difference in radiation dose between both compression paddles.

For the $\mathrm{CC}$ view, the mean posterior nipple line length was $10.9 \mathrm{~cm}(\mathrm{sem} \pm 0.2 \mathrm{~cm}, p=0.71)$ for both compression paddles (see Table 1); there was also no difference between FP and RP according to paddle size. For the MLO view, the posterior

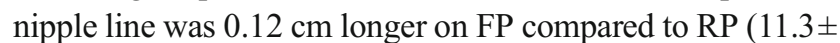
$0.2 ; 11.2 \pm 0.2$ respectively; $p=0.00$ ), which was due to the effect observed with the large paddle $(24 \times 29 \mathrm{~cm})$. The percentage difference observed in all comparisons for this 


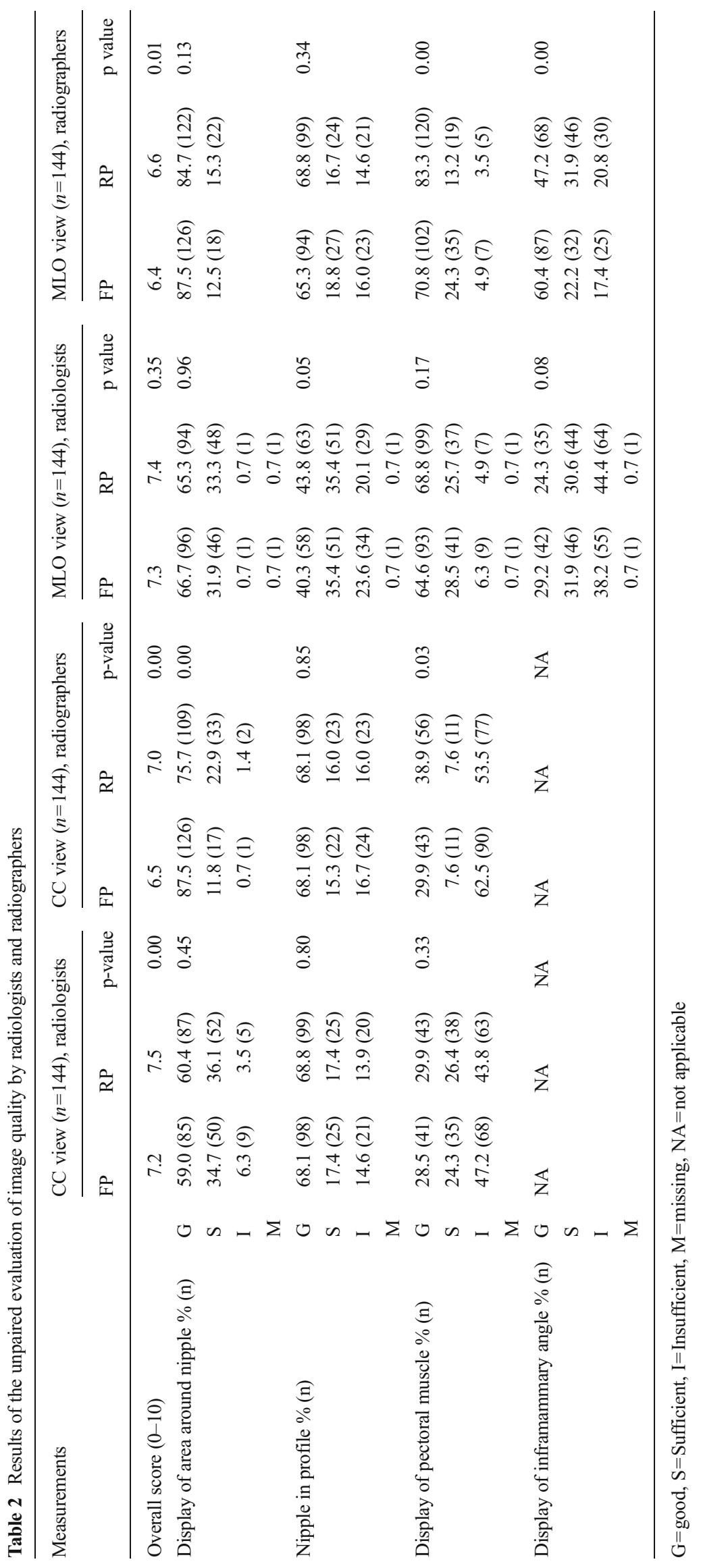




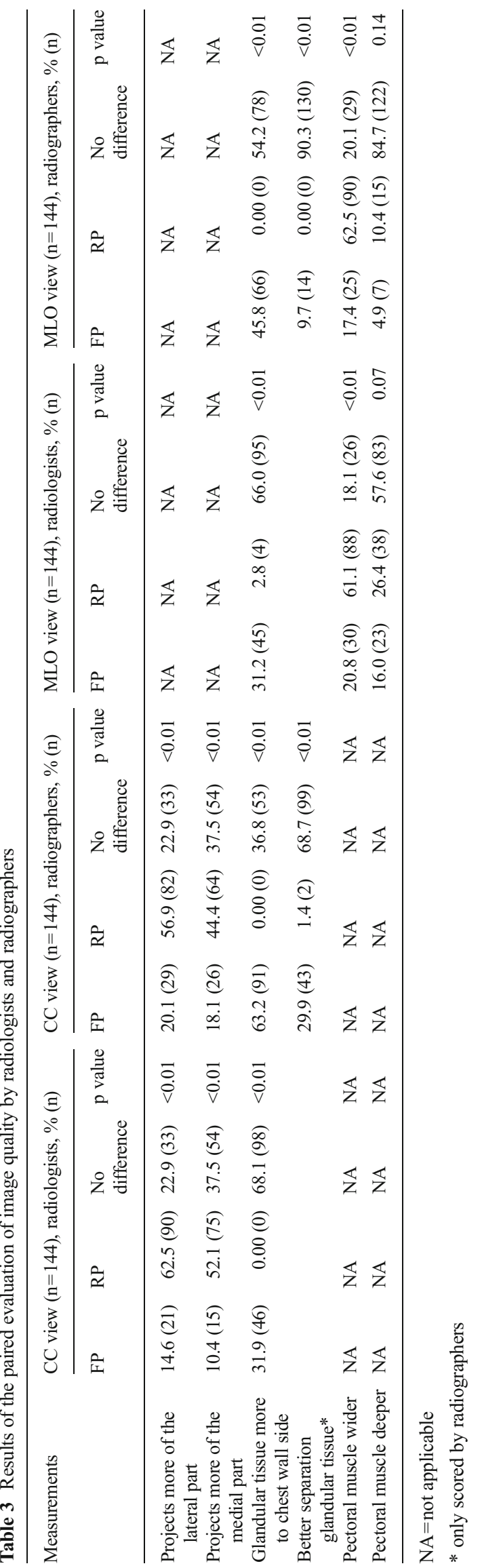

distance parameter ranged from $-0.12 \%$ to $1.58 \%$. The mean projected breast area on the mammogram is larger when using FP, which was also due to the differences in the large paddle. For the CC view, FP resulted in $180.0 \pm 5.7 \mathrm{~cm}^{2}$ and RP in $178.6 \pm 5.6 \mathrm{~cm}^{2}(p=0.11)$; for the MLO view, the area depicted was $218.7 \pm 5.5 \mathrm{~cm}^{2}$ and $217.0 \pm 5.3 \mathrm{~cm}^{2}$, respectively ( $p=$ 0.03 ). The percentage difference between FP and RP for the mean projected breast area in the comparisons ranged from $0.29 \%$ to $1.50 \%$.

Results of the evaluation of technical image quality are presented in Tables 2 (unpaired evaluation) and 3 (paired evaluation). The overall score for image quality in the unpaired evaluation was higher for RP than FP in both views and for both radiologists and radiographers; the difference was more prominent in the CC view ( $p=0.00$; Table 2$)$. The area around the nipple was well imaged with both paddles, although radiographers rated a significantly higher proportion of images with FP as 'good' on the CC view ( $87.5 \%$ vs $75.7 \%$; $p=0.00$ ). FP further showed an improved display of the inframammary angle on the MLO view compared to RP, particularly in the scores of the radiographers $(p=0.00)$. RP showed an increased free projection of the nipple at MLO view compared to FP only according to the radiologists ( $p=0.05$ ), and an increased display of the pectoralis muscle for both views (CC $p=0.03$, MLO $p=$ 0.00 ) only according to the radiographers.

In the paired evaluation, all observers found that RP showed significantly more of the lateral and medial part of the breast in the $\mathrm{CC}$ view and projected the pectoral muscle wider on the MLO view (Table 3). They also agreed that FP projected the glandular tissue more to chest wall side than RP, with a more prominent difference noted by the radiographers. In addition, the radiographers found that separation of breast tissue was mostly equal but otherwise more often improved with FP. Figure 2a-c show illustrations of the above-mentioned results (more examples are provided in a supplementary video, available online).

An accessory finding that was reported by all observers in the open comment boxes was that contrast in the retroglandular area was considerably less with FP than RP. To gain support for this finding, we asked an independent radiologist to score the images on contrast in the retroglandular area. This radiologist was blinded for the paddle used and was not informed about the remarks of the other observers. The results showed that RP had a better contrast in $80.1 \%$ of the cases than FP; FP showed a better contrast in $9.4 \%$ of the cases and no difference in contrast was found in $10.5 \%$ of the cases. The results were comparable for the CC and MLO views.

\section{Discussion}

In this first study on clinical performance of breast compression paddles, we found no differences in pain experience for 

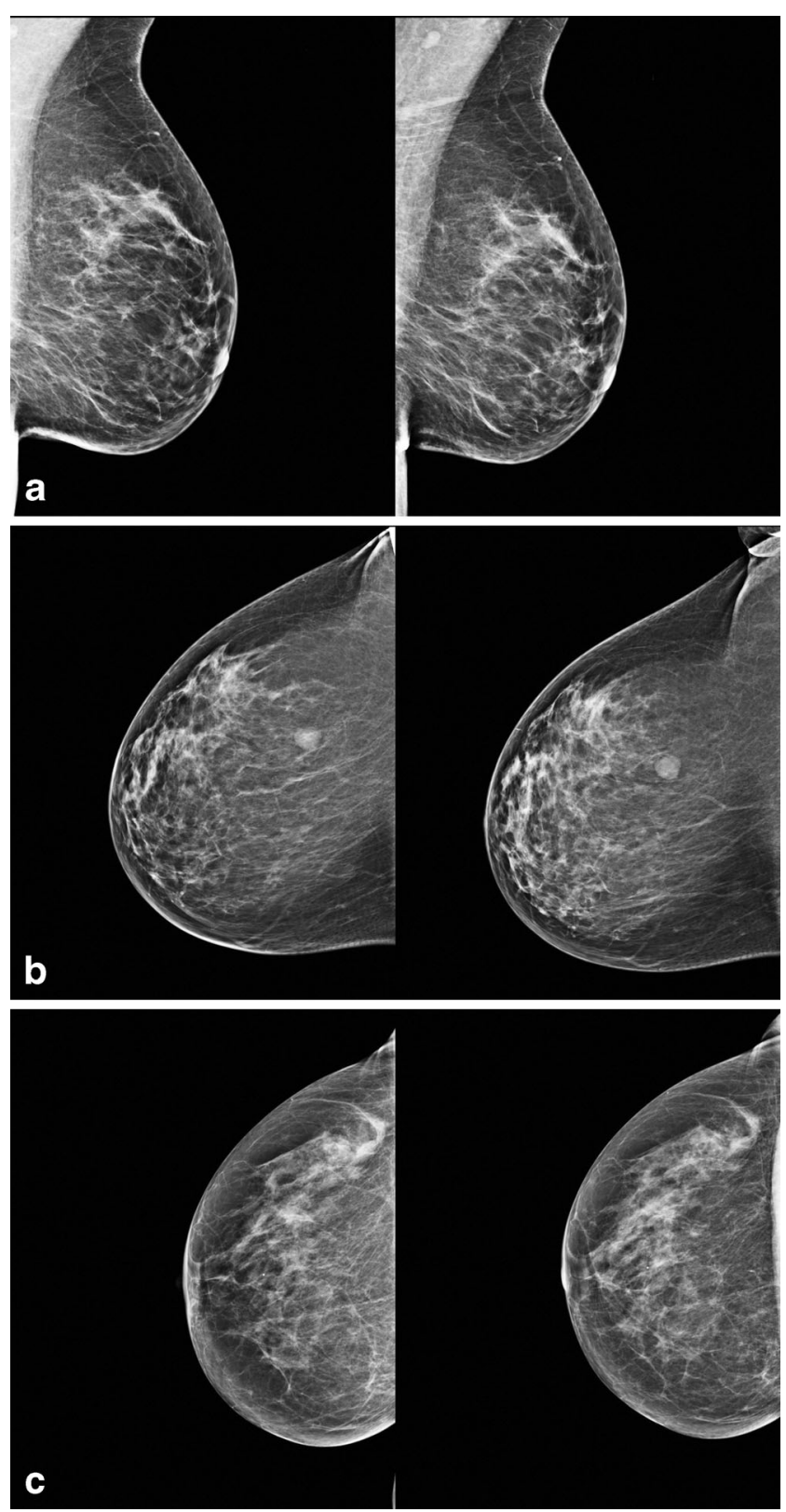

Fig. 2 a MLO images of the right breast in one client with FP (left $)$ and $\mathrm{RP}($ right). With FP, the inframammary angle is better displayed; with RP, the pectoralis muscle is better imaged. $\mathbf{b}$ CC images of the right breast in one client with FP (left) and RP (right). With FP, the breast tissue is moved towards the chest wall side, thereby pushing some part of the retroglandular tissue off the bucky. $\mathbf{c} \mathrm{CC}$ images of the right breast in one client with FP (left) and RP (right). With FP, the retroglandular area is smaller and less well depicted, due to a loss of contrast

FP compared to RP in our study setting. However, we found that RP depicted more breast tissue and showed better contrast than FP, especially in the retroglandular area. With FP, areas of fibroglandular tissue were pushed out of the image area at the chest wall side, as shown in Figs. 1 and 2. This threedimensional effect can only be noted through paired evaluation of technical image quality using the toggle (blink) mode. FP even performed slightly better in the two-dimensional projection of the posterior nipple line and projected breast area. However, these latter differences were very small, in the range of $-0.29 \%$ to $1.58 \%$, and not considered clinically relevant. We further calculated a slightly lower radiation dose with FP ( $4.5 \%$ of mean dose), but this finding has to be interpreted with care, given the uncertainties in these estimates (see Material and Methods section).

The decreased contrast of FP at the retroglandular area is a consequence of a different distribution of light and dark areas between FP and RP. At nipple side the images with FP are darker than RP, and at the retroglandular area, the images with FP are lighter than with RP. The lighter retroglandular area with FP results in a lower perceived contrast compared to RP (see also Fig. 2c).

We were not able to confirm increased comfort for the women with FP, as suggested by some manufacturers at the introduction of flexible compression paddles. This might be partly explained by the higher compression force applied in the Netherlands $(12.8 \mathrm{daN})$ than in other countries $[14,15]$. Both in the paired and unpaired evaluation of technical image quality, we found that differences between the two paddles were mostly more prominent in the scores of radiographers, even though the direction of the difference was usually the same for both groups of observers. This could be explained by the fact that radiographers were more focused on optimal positioning, whereas radiologists were more likely focusing on whether the images were sufficient for diagnostic evaluation.

To our knowledge, only one previous study compared image quality and radiographer performance for two different compression paddles [7]. In this study, Sardanelli et al. showed that biphasic compression outperformed monophasic compression. However, although monophasic compression was similar to RP in our study, the tilt in biphasic compression was opposite to FP: compression started with an angled paddle of $22.5^{\circ}$ downward and ended when the paddle was parallel to the cassette holder. Therefore, the results of this study cannot be compared directly to our study.

The longer mean posterior nipple line and larger projected breast area with FP seem to contradict the subjective evaluation of technical image quality that was in favour of RP. With $\mathrm{FP}$, breast tissue is moved from the image area at chest wall side. We hypothesize that this effect is due to the tilt angle of FP, which decomposes the applied force into a component perpendicular to the detector, which is effective for compression, and a component parallel to the detector, which is lost in pushing the breast away. The ratio of lost and effective force depends on the tilt angle. During the second part of compression with FP, the paddle tilts towards nipple side, which pushes the upper part of the breast and breast tissue backwards. However, because the skin and the lower part of the breast remain fixed on the bucky, the projected breast area for FP is not smaller compared to RP. On the contrary, the nipple 
side will be compressed more with FP, which can explain the small increases in posterior nipple line and projected breast area for FP compared to RP. For future comparisons of different paddles, it would be advisable to estimate breast volume instead of using two-dimensional measures as a proxy.

For the determination of radiation dose, a correct measure of breast thickness is needed. With FP, breast thickness decreases towards the nipple side, resulting in a wedge-shaped rather than a flat breast, which means that the breast dosimetry model cannot be properly applied. Dose delivery in a wedge-shaped breast will be different and would ideally require a more complex model. In our study, we found that radiation dose based on the model by Dance et al. was somewhat lower with FP compared to RP. However, the difference was small, only $4.5 \%$, and similar to the acceptable variation in radiation dose with respect to the stability of the mammographic unit. Had we used the radiation dose from the DICOM-header, this would have resulted in an equally small difference of $2.5 \%$ between the two paddle types. Nevertheless, firm conclusions about differences in radiation dose are not possible.

A limitation of this study is that a structural positioning difference between RP and FP could have been introduced. This study was designed as a prospective cross-over trial with each woman serving as her own comparison. The mammographic screening examination was obtained in a routine fashion with FP except that each woman received an additional image with RP for one view, with the order of the paddles randomly assigned for the selected view. It was not possible to blind the radiographers to the paddle used, but they did receive an instruction on the importance of working objectively in order to minimize variation in positioning. In addition, we involved only three experienced radiographers who were skilled in the use of both paddles. Despite these efforts, there could still be some variation in positioning of the breast. However, due to the very small differences in the posterior nipple line distance and the projected breast area between FP and $\mathrm{RP}$, we are confident that a structural positioning difference between the two paddles did not appear.

In summary, pain experience showed no difference between the two paddles. Overall, FP performed slightly better in the area of breast tissue projected, whereas RP performed better in the paired evaluation of technical image quality. However, the finding that FP removes breast tissue from the image area at chest wall side weighs heavily in our overall comparison. Further, to our knowledge, this is the first study to report that contrast in the retroglandular area is superior with RP. This area represents a clinically relevant part of the breast for the detection of cancer [16, 17]. A similar problem has been reported in relation to breast density measurements, where the conclusion was that software needs a correction when FP is used $[18,19]$. On balance, we therefore recommend the use of the RP for standard mediolateral-oblique and craniocaudal views.

Acknowledgments The authors wish to thank Michiel Kallenberg, Nico Karssemeijer and Jerry de Groot for advice on estimating breast volume from mammographic images recorded with a flexible compression paddle.

The scientific guarantor of this publication is M.J.M. Broeders, Dutch Reference Centre for Screening. The authors of this manuscript declare relationships with the following companies: G.J.H.: Founder (but not share holder) of Sigmascreening, a spin-off company of $t$ the Academic Medical Center, Amsterdam. The authors state that this work has not received any funding. No complex statistical methods were necessary for this paper. Institutional Review Board approval was not required because a permit for this study, equal to institutional review board approval, was obtained from the Minister of Health, Welfare and Sport under the Dutch Population Screening Act in October 2010. Written informed consent was obtained from all subjects (patients) in this study. Methodology: prospective, randomised controlled trial, performed at one institution.

Open Access This article is distributed under the terms of the Creative Commons Attribution Noncommercial License which permits any noncommercial use, distribution, and reproduction in any medium, provided the original author(s) and the source are credited.

\section{References}

1. Perry N, Broeders M, de WolfC, Törnberg S, Holland R, von Karsa L (2006) European guidelines for quality assurance in breast cancer screening and diagnosis, 4th edn. Office for Official Publications of the European Communities, Luxembourg

2. Drossaert CHC, Boer H, Seydel ER (2002) Monitoring women's experiences during three rounds of breast cancer screening: results from a longitudinal study. J Med Screen 9:168-175

3. Keemers-Gels ME, Groenendijk RPR, van den Heuvel JHM, Boetes C, Peer PGM, Wobbes T (2002) Pain experienced by women attending breast cancer screening. Breast Cancer Res Treat 60:235-240

4. Myklebust AM, Seierstad T, Stranden E, Lerdal A (2009) Level of satisfaction during mammography screening in relation to discomfort, service provided, level of pain and breast compression. Eur J Radiogr 1:66-72

5. Miller D, Livingstone V, Herbison P (2008) Interventions for relieving the pain and discomfort of screening mammography. Cochrane Database Syst Rev Issue 1. CD002942

6. Geeraert N, Klausz R, Lemuhof M, Sundermann D, Muller S (2011) Contribution of compression paddle flexibility to estimation of breast glandularity. ECR 2011 Book of Abstracts. Insights Imaging 2(Suppl 1):B-274

7. Sardanelli F, Zandrino F, Imperiale A, Bonaldo E, Quartini MG, Cogorno N (2000) Breast biphasic compression versus standard monophasic compression in X-ray mammography. Radiology 217: 576-580

8. Hauge IHR, Hogg P, Szczepura K, Connolly P, McGill G, Mercer C (2012) The readout thickness versus the measured thickness for a range of screen film mammography and full-field digital mammography units. Med Phys 39:263-271 
9. National Evaluation Team for Breast Cancer Screening (2011) NETB Interim Report. Main results 2008-2009 breast cancer screening programme in the Netherlands. 2011. Rotterdam, Dept. of Public Health, Erasmus MC, University Medical Center Rotterdam

10. Williamson A, Hoggart B (2005) Pain: a review of three commonly used pain rating scales. J Clin Nurs 14:798-804

11. Eklund GW, Cardenosa G (1992) The art of mammographic positioning. Radiol Clin N Am 30:21-53

12. Bassett L (1995) Clinical image evaluation. Radiol Clin N Am 33: $1027-1039$

13. Dance DR, Skinner CL, Young KC, Beckett JR, Kotre CJ (2000) Additional factors for the estimation of mean glandular breast dose using the UK mammography dosimetry protocol. Phys Med Biol 45: 3225-3240

14. Dustler M, Andersson I, Brorson H et al (2012) Breast compression in mammography: pressure distribution patterns. Acta Radiol 53: 973-980
15. Hendrick RE, Pisano ED, Averbukh A et al (2010) Comparison of acquisition parameters and breast dose in digital mammography and screen-film mammography in the American College of Radiology Imaging Network digital mammographic imaging screening trial. AJR Am J Roentgenol 194:362-369

16. Tabár L, Dean PB (1985) Tabar's forbidden zones. In: Tabár L, Dean PB (eds) Teaching atlas of mammography, 2nd edn. Thieme Inc, New York, pp 5-14

17. Brown M, Eccles C, Wallis MG (2001) Geographical distribution of breast cancers on the mammogram: an interval cancer analysis. $\mathrm{Br} \mathrm{J}$ Radiol 74:317-322

18. Kallenberg MG, Van Gils CH, Lokate M, den Heeten GJ, Karssemeijer N (2012) Effect of compression paddle tilt correction on volumetric breast density estimation. Phys Med Biol 57:5155-5168

19. Kallenberg MG, Karssemeijer N (2012) Compression paddle tilt correction in full-field digital mammograms. Phys Med Biol 57: $703-715$ 